\title{
The Testing of \\ Causal Stock Returns-Trading Volume Dependencies with the Aid of Copulas
}

\section{Introduction}

Market participants usually think that a share price reflects investors' predictions about the future performance of a company. These expectations are based on available information about the company. The release of new information forces investors to change their expectations about the future performance of the company. New announcements are the main source for price fluctuations. Since investors evaluate the content of new information differently, prices may remain constant even though new information is important for the market. This can be the case if some investors think that the news is good, whereas others understand the same announcements quite differently. The direction of movements of prices depends on the average reaction of investors to news.

It is obvious that share prices can be observed if there is a positive trading volume. As with prices, trading volume and its changes react to the available set of important information on the market. Trading volume reacts in a different way in comparison to stock prices. A change in investors' expectations always leads to a rise in trading volume. The trading volume adjusts the sum of investors' reactions to news.

A real answer to the question whether a knowledge of one variable (e.g. volatility) can improve short-run forecasts of other variables is essential not only for analysts but also for market participants. Thus, in recent years both researchers

* AGH University of Science and Technology in Cracow, Department of Applications of Mathematics in Economics, e-mail: henryk.gurgul@gmail.com.

Financial support for this paper from the National Science Centre of Poland (Research Grant DEC2012/05/B/HS4/00810) is gratefully acknowledged.

** University of Applied Sciences Joanneum in Graz, Department of Banking and Insurance, e-mail: roland.mestel@uni-graz.at

*** Jagiellonian University in Cracow, Institute of Economics and Management, e-mail: robert.syrek@uj.edu.pl 
and investors have focused on the links between trading volume, stock return and return volatility. Most early empirical examinations have been concerned the contemporaneous relationship between price changes and trading volume.

Both from a theoretical and practical point of view the dynamic relationship between returns, return volatility and trading volume is more interesting than the contemporaneous one. One of the most important and useful topics in empirical economics is an examination of the causal relationship between the variables in question. The notion of causality was introduced by Granger [20]. It is based on the idea that the past cannot be determined by the present or future. Thus, if one event is observed before another event, causality can only take place from the first event to the second one.

Brief information about some aspects of causality and the background literature contributions will be given in the next section. The concepts of nonlinear causality and Bernstein copulas are outlined in the third section. Data description and the estimation method of realized volatility are presented in the fourth section. The empirical results are discussed in the fifth section. Brief conclusions and outlook are given in the last part of the paper.

\section{Literature review}

Karpoff [30] in his survey of early research about price-volume relations citied important reasons for regarding price-volume dependencies. This permits $\mathrm{zn}$ insight into the structures of financial markets, and an understanding of the information arrival process especially how information is disseminated among market participants. It is strictly related to two hypotheses: the mixture of distributions hypothesis (MDH), Clark [13], Epps and Epps [17], Tauchen and Pitts [44] and Harris [28] and the sequential information arrival hypothesis by Copeland [14] and Tauchen and Pitts [44]. The knowledge of price-volume relations is also useful in technical analysis and it is important with respect to investigations of options and futures markets and in fashioning new contracts.

One of the most useful approaches in the research concerning return-trading volume interrelations is the concept of Granger causality [20]. Causality in the Granger sense can be understood as a kind of conditional dependency. Using this causality methodology we can check if the past values of one stationary variable is helpful in predicting the future values of another one or not. In most early papers concerned with causal dependencies linear vector autoregression models are used. The significance of coefficient estimates of a potentially causal variable means the existence of causality running from this variable to the endogenous variable. However, in the case of the nonstationarity of the time series under study 
to causality can be spurious (comp. Granger and Newbold [21], Phillips [38]). In the case of cointegration VECM can be applied for causality testing. If one finds the respective coefficient of error correction to be not-negative, then it can be concluded that there is no stable and long-run relationship.

Another serious problem concerning the linear approach to testing for causality is the low power of the tests needed to detect some kinds of nonlinear causal relationships. This problem is raised in contributions which are concerned with nonlinear causality tests (see e.g. Abhyankar [1] and Asimakopoulos [2]). The starting point for further investigations was the nonparametric statistical method for uncovering nonlinear causal effects presented by Baek and Brock [7]. In order to detect causal relations the authors used the correlation integral, an estimator of spatial probabilities across time based upon the closeness of points in hyperspace.

The concept by Hiemstra and Jones [29] improves the small-sample properties of the causality test and relaxes the assumption that the series to which the test is applied are i.i.d. The authors conducted some Monte Carlo simulations and proved the robustness of their test for the presence of structural breaks in the series. They also checked contemporaneous correlations in the errors of the VAR model in order to filter out linear cross- and auto-dependence.

Diks and Panchenko [15, 16] noticed the fact that the null hypothesis in the $\mathrm{HJ}$ (Hiemstra and Jones) test is generally not equivalent to Granger non-causality. The authors developed their own test with better performance than the $\mathrm{HJ}$ one, especially in terms of over-rejection and size distortion, which are frequently reported for the $\mathrm{HJ}$ test. The authors gave some recommendations, including bandwidth adaptation for ARCH type processes. In this case, the optimal bandwidth can be expressed in terms of the ARCH model coefficient.

There are also a few papers e.g. Sims et al. [41], Toda and Yamamoto [45] which demonstrated that the asymptotic distribution theory is not a proper basis for testing the causality of integrated variables by mean of the VAR model. This also holds true in a case where the variables are cointegrated. Therefore, an alternative concept of causality testing was developed based on a Wald test statistic.

Almost all of the papers analyzed the dynamic links of indexes and individual companies listed in these indexes from highly developed stock markets.

Gurgul et al. [25] investigated the causal relationships between trading volume and stock returns and return volatility for the Warsaw Stock Exchange. Applying the linear Granger causality test, the contributors observed a significant causal relationship between returns and trading volume in both directions and linear Granger causality from return volatility to trading volume. In addition, their findings showed that a knowledge of past stock price movements on the German as well as the US stock market supported short-run predictions of current and future trading volume of the companies from the WSE. 
Gurgul et al. [23] in a contribution based only on the traditional linear approach concluded that short-run forecasts of current or future stock returns cannot be improved by a knowledge of recent volume data and vice versa.

The linear and nonlinear causality of companies listed in, DAX index was investigated by Gurgul and Lach [22]. They used daily data at close from the time period January 2001- November 2008. The contributors presented the results of linear and nonlinear testing for causality. While for testing of the nonlinear causality the Diks and Panchenko test was used the linear dependencies were checked by traditional Vector Autoregression Models and by the model derived by Lee and Rui [31]. The contributors confirmed the hypothesis that traditional linear causality tests often fail to detect some kinds of nonlinear relations, while nonlinear tests do not. In many cases the test results obtained by use of empirical data and simulation confirmed a bidirectional causal relationship while a linear test did not detect such causality at all.

A hypothesis on dynamic interdependencies between returns and trading volume is the bigh volume premium bypothesis. High trading volume of a stock implies that investors focus on that stock. This implies that such a stock seems to be more interesting to potential investors which is whythe stock prices tend to increase. This hypothesis was tested by Gervais et al. [19]. The contributors checked this hypothesis for companies listed on the NYSE. The results are in line with the bigh volume premium bypothesis since the authors observed that high trading volume precedes high returns while small trading volume precedes low returns.

Gurgul and Wójtowicz [25], taking into account event study methodology defined an event as the appearance of extreme high trading volume. The authors tested the high volume premium hypothesis for companies listed on the WSE. The results were in line with the high volume premium conjecture since the occurrence of high trading volume implied high returns (especially in the case of small companies) in the following days, especially one day after. The results not only supported the high trading volume premium hypothesis but also suggested the construction of profitable investment strategies. In addition, in the case of small trading volume the mean abnormal returns were not statistically significant.

The papers above were concerned with dynamic dependencies between returns, return volatility and trading volume on the basis of daily data. However, these dependencies are much more interesting for high frequency data. In all papers reviewed absolute values or squared stock returns were applied as proxies for volatility. There are few papers dealing with intraday data when links between returns, return volatility and trading volume are examined.

Rossi and Magistris [40] investigated the relationship between realized volatility and trading volume. They showed that volume and volatility exhibit long 
The Testing of Causal Stock Returns-Trading Volume Dependencies...

memory but they are not driven by the same latent factor as suggested by fractional cointegration analysis. They used the fractional cointegration VAR models of Nielsen and Shimotsu [37], which extend the analysis of Robinson and Yajima [39] to stationary and nonstationary time series as well. They found that past (filtered) log-volume has a positive effect on current filtered log-volatility and current log-volume as well. Their analysis was complemented by using copulas in order to measure the degree of tail dependence. The series of log-volume and log-volatility are found to be dependent in extreme values. Luu and Martens [33] conducted some tests of the mixture of distributions hypothesis using realized volatility and found bidirectional causality of realized volatility and the trading volume of S\&P500 index future contracts, whereas when using daily stock returns the MDH was not supported. The results of long memory analysis suggested that trading volume and volatility share the same degree of long-run dynamics, which supported the Bollerslev and Jubinski [11] version of $\mathrm{MDH}$. Fleming and Kirby [18] also considered the Bollerslev and Jubinski [11] interpretation of MDH but they used fractionally-integrated time series models to investigate the joint dynamics of trading volume and volatility. The contributors examined this issue using more precise volatility estimates obtained using high-frequency returns (i.e. realized volatilities). Their results indicated that both volume and volatility displayed long memory. However they rejected the hypothesis that the two series shared a common order of fractional integration for a fifth of the firms in their sample. Moreover, the authors found a strong correlation between innovations in volume and volatility. The contributors draw the conclusion that trading volume can be used to obtain more precise estimates of daily volatility for cases in which high-frequency returns are unavailable. Bouezmarni et al. [12] derived a nonparametric test based on Bernstein copulas and tested on the basis of high frequency data for causality between stock returns and trading volume. The contributors proved, that at a 5\% significance level, the nonparametric test rejected clearly the null hypothesis of non-causality from returns to volume, which is in line with the conclusion which followed from the linear test. Further, their nonparametric test also detects a non-linear feedback effect from trading volume to returns at a 5\% significance level.

In the next part of this paper in order to check links between the financial variables under study realized volatility will be used as a proxy for volatility.

\section{Nonlinear causality and Bernstein copulas}

Now, we will present an extension of the Granger causality notion taking into account three variables $X, Y$ and $Z$. Variable $Z$ is in a causal relation to variable $Y$ 
in the Granger sense if the current values of the variable $Y$ can be forecasted more precisely by means of the known past values of variable $Z$, and those of auxiliary variable $X$, than in the case where the values of variable $Z$ are not involved in the forecasting process.

In the recent literature on nonlinear dependencies in the sense of Granger causality nonparametric tests are used for the conditional independence of random variables. The conditional independence of random variables implies a lack of causality in the Granger sense. Linton and Gozalo [32] tested conditional independence by mean of a test statistic based on empirical distributions. Su and White [42] derived a test based on smoothed empirical likelihood functions and in the year 2007 developed a nonparametric test for the conditional independence of distributions. To this end, they applied conditional characteristic functions. The test for conditional independence by $\mathrm{Su}$ and White [43] is based on a kernel estimation of conditional distributions $f(y \mid x)$ and $f(y \mid x, z)$ if the null holds true than the last functions are equal. The serious drawback of this test is the restriction of the sum of dimensions of variables $X, Y, Z$ to seven. In addition, it is necessary to define a weight function for Hellinger distance which is needed to measure the distance between the conditional distributions. The contributors applied their test to examine Granger non-causality in exchange rates. These drawbacks were addressed by Bouezmarni et al. [12]. We use their approach and methodology in the empirical part of this paper. The causality test applied for the detection of nonlinear causality is based on Bernstein copulas, which are presented in the next section.

\subsection{Bernstein copulas}

The estimator of density of a Bernstein copula in $g=\left(g_{1}, g_{2}, g_{3}\right)$ is determined by the expression:

$$
\hat{c}_{X Y Z}\left(g_{1}, g_{2}, g_{3}\right)=\frac{1}{T} \sum_{t=1}^{T} K_{k}\left(g, \hat{G}_{t}\right),
$$

where $\hat{G}_{t}=\left(\hat{G}_{t 1}, \hat{G}_{t 2}, \hat{G}_{t 3}\right)$ stands for the vector $\left(\hat{F}_{X}\left(X_{t}\right), \hat{F}_{Y}\left(Y_{t}\right), \hat{F}_{Z}\left(Z_{t}\right)\right)$.

The kernel $K_{k}=\left(g, \hat{G}_{t}\right)$ can be calculated by use of the binomial distributions:

$$
p_{v_{j}}\left(g_{j}\right)=\left(\begin{array}{c}
k-1 \\
v_{j}
\end{array}\right) g_{j}^{v_{j}}\left(1-g_{j}\right)^{k-v_{j}-1}
$$

for $v_{j}=0, \ldots, k-1$ and $j=1,2,3$. 
Assume $A_{\hat{G}_{t}, v}=1_{\left\{\hat{G}_{t} \in B_{v}\right\}}$ to be the characteristic (indicator) function of set $B_{V}$ which is defined as

$$
B_{v}=\left[\frac{v_{1}}{k}, \frac{v_{1}+1}{k}\right] \times\left[\frac{v_{2}}{k}, \frac{v_{2}+1}{k}\right] \times\left[\frac{v_{3}}{k}, \frac{v_{3}+1}{k}\right] .
$$

The kernel can be written as

$$
K_{k}\left(g, \hat{G}_{t}\right)=k^{3} \sum_{v_{1}=0}^{k-1} \sum_{v_{2}=0}^{k-1} \sum_{v_{3}=0}^{k-1} A_{\hat{G}_{t}, v} \prod_{j=1}^{3} p_{v_{j}}\left(g_{j}\right)
$$

One can estimate a kernel $K_{k}\left(g, \hat{G}_{t}\right)$ by the use of the density function of Beta distributions with parameters $v_{j}+1$ and $k-v_{j}$ :

$$
K_{k}\left(g, \hat{G}_{t}\right)=\sum_{v_{1}=0}^{k-1} \sum_{v_{2}=0}^{k-1} \sum_{v_{3}=0}^{k-1} A_{\hat{G}_{t}, v} \prod_{j=1}^{3} B\left(g_{j}, v_{j}+1, k-v_{j}\right)
$$

In the case of a two-dimensional copula the last expression for variables $X$ and $Y$ is given by

$$
\hat{c}_{X Y}\left(g_{1}, g_{2}\right)=\frac{1}{T} \sum_{t=1}^{T} K_{k}\left(g, \hat{G}_{t}\right),
$$

where $\hat{G}_{t}=\left(\hat{G}_{t 1}, \hat{G}_{t 2}\right)=\left(\hat{F}_{X}\left(X_{t}\right), \hat{F}_{Y}\left(Y_{t}\right)\right)$.

In addition

$$
B_{v}=\left[\frac{v_{1}}{k}, \frac{v_{1}+1}{k}\right] \times\left[\frac{v_{2}}{k}, \frac{v_{2}+1}{k}\right]
$$

and

$$
K_{k}\left(g, \hat{G}_{t}\right)=k^{2} \sum_{v_{1}=0}^{k-1} \sum_{v_{2}=0}^{k-1} A_{\hat{G}_{t}, v} \prod_{j=1}^{2} p_{v_{j}}\left(g_{j}\right)=\sum_{v_{1}=0}^{k-1} \sum_{v_{2}=0}^{k-1} A_{\hat{G}_{t}, v} \prod_{j=1}^{2} B\left(g_{j}, v_{j}+1, k-v_{j}\right) .
$$

In the above equation the number $k$ is the only parameter which should be set before the computations. This parameter stands for the number of ,picks” of two dimensional distribution for which the density function is smoothed. It is obvious that the accuracy tends to increase as $k$ rises. Figure 1 demonstrates a method for the nonparametric estimation of a Gumbel copula by use of a Bernstein copula. Therefore, 1000 realizations from a Gumbel copula with parameter 2 were generated. Next for $k=\lfloor 2 \sqrt{1000}\rfloor=63$ the density function*, was estimated by means of a nonparametric estimator based on binomial distributions.

* $\lfloor x\rfloor$ denotes the integer part of the real number $x$. 

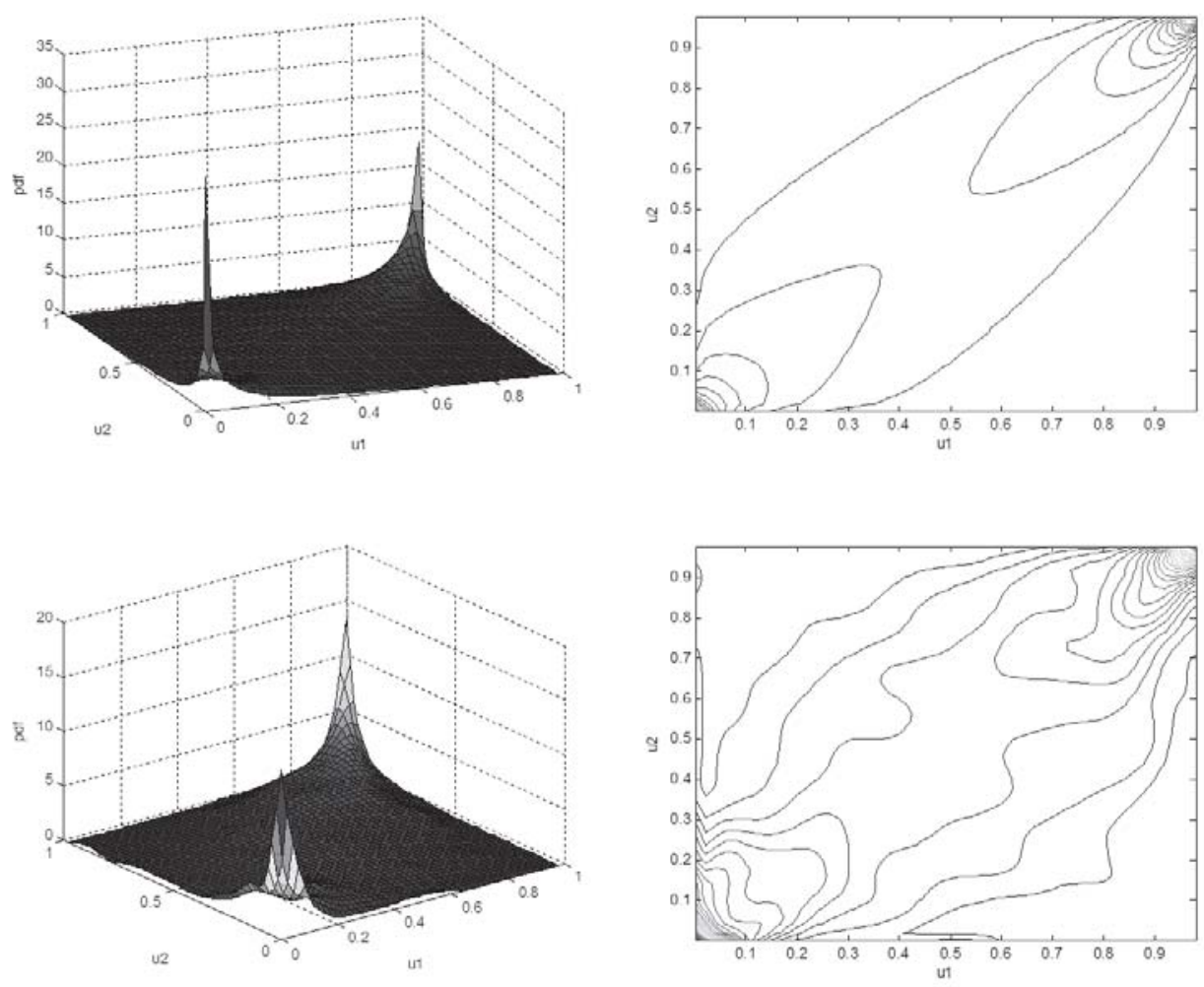

Figure 1. The density of the Gumbel copula and its contours (upper plots). Below, the density of the Bernstein copula and its contours.

Source: own elaboration

Bouezmarni et al. [12] focus on the differences between their test (called hereafter the BRT test) and the test by Su and White [43]. The main differences (and advantages of the BRT test) can be summarized as follows:

1. There is no restriction on the sum of dimensions of variables under study.

2. The application of a nonparametric Bernstein copula in order to estimate the joint conditional distributions guarantees the non-negativity of their distributions. This is important with respect to the proper determination of the distance between them by means of the Hellinger distance.

3. It is necessary to determine only one parameter which determines the accuracy of the estimation of nonparametric copula density. 
The contributors demonstrated by means of simulation studies that their test has appropriate power and allows a recognition of different nonlinear dependencies between variables. By means of simulation exercises they also supplied evidence for the uselessness of a classic linear causality test for the detection of causal dependencies between nonlinear processes. The authors applied their test in an examination Granger non-causality between many macroeconomic and financial variables.

\subsection{Nonlinear causality versus conditional dependence}

Let $\left\{\left(X_{t}^{\prime}, Y_{t}^{\prime}, Z_{t}^{\prime}\right) \in \mathbb{R}^{d_{1}} \times \mathbb{R}^{d_{2}} \times \mathbb{R}^{d_{3}}, t=1 \ldots T\right\}$ be a realization of the stochastic process in $\mathbb{R}^{d}$, where $d=d_{1}+d_{2}+d_{3}$ with joint distribution $F_{X Y Z}$ and density function $f_{X Y Z}$. The test of conditional independence between variables $Y$ and $Z$ under condition $X$ can be written down for density functions as (Bouezmarni et al. [12]):

$$
\begin{gathered}
H_{0}: P\left(f_{Y \mid X, Z}(y \mid x, z)=f_{Y \mid X}(y \mid x)\right)=1, \forall y \in \mathbb{R}^{d_{2}}, \\
H_{1}: P\left(f_{Y \mid X, Z}(y \mid x, z)=f_{Y \mid X}(y \mid x)\right)<1, \text { for } y \in \mathbb{R}^{d_{2}},
\end{gathered}
$$

where $f_{\cdot \mid}(\cdot \cdot)$ stands for the conditional density function.

It is worth noting that a lack of causality in the Granger sense can be understood as conditional independence. Let $(Y, Z)^{\prime}$ be a Markov process of order 1 . Variable $Z$ does not cause variable $Y$ in the Granger sense if and only if the following null hypothesis holds true:

$$
H_{0}: P\left(f_{Y \mid X, Z}\left(y_{t} \mid y_{t-1}, z_{t-1}\right)=f_{Y \mid X}\left(y_{t} \mid y_{t-1}\right)\right)=1,
$$

i.e. $y=y_{t}, x=y_{t-1}, z=z_{t-1}$, for $d_{1}=d_{2}=d_{3}=1$.

For the sake of simplicity of notation we assume $d_{i}=1$ for $i=1,2,3$. Taking into account this notation the well-known Sklar theorem can be put in the form:

$$
F_{X Y Z}(x, y, z)=C_{X Y Z}\left(F_{X}(x), F_{Y}(y), F_{Z}(z)\right) .
$$

The respective density function $f_{X Y Z}$ is given by the equation

$$
f_{X Y Z}(x, y, z)=f_{X}(x) f_{Y}(y) f_{Z}(z) c_{X Y Z}\left(F_{X}(x), F_{Y}(y), F_{Z}(z)\right),
$$

where $c_{X Y Z}$ is the density function of copula $C_{X Y Z}$. 
The null hypothesis (1) can be expressed by means of the copula notion in the following form:

$$
H_{0}: P\left(c_{X Y Z}\left(F_{X}(x), F_{Y}(y), F_{Z}(z)\right)=c_{X Y}\left(F_{X}(x), F_{Y}(y)\right) c_{X Z}\left(F_{X}(x), F_{Z}(z)\right)\right)=1, \forall y \in \mathbb{R}
$$

while alternative hypothesis (2) fulfills the inequality:

$$
H_{1}: P\left(c_{X Y Z}\left(F_{X}(x), F_{Y}(y), F_{Z}(z)\right)=c_{X Y}\left(F_{X}(x), F_{Y}(y)\right) c_{X Z}\left(F_{X}(x), F_{Z}(z)\right)\right)<1
$$

for $y \in \mathbb{R}$, where $c_{X Y}$ and $c_{X Z}$ stand for densities of copulas of two dimensional distributions $(X, Y)$ and $(X, Z)$. The test statistics suggested by Bouezmarni et al. (2012) are based on the Hellinger distance between two distributions i.e. the density of the copula $c_{X Y Z}$ and the product of the densities of copulas $c_{X Y}$ and $c_{X Z}$. This measure

$$
H(c, C)=\int_{[0,1]^{3}}\left(1-\sqrt{\frac{c_{X Y}(u, v) c_{X Z}(u, w)}{c_{X Y Z}\left(F_{X}(x), F_{Y}(y), F_{Z}(z)\right)}}\right)^{2} d C_{X Y Z}(u, v, w) .
$$

is equal to zero if the null hypothesis holds true.

The distance (3) exhibits important advantages. First of all, it is symmetric and invariant with respect to monotone transformations. In addition, it is not sensitive to outliers, because their weights are lower than the weights of other observations. For empirical data the Hellinger distance (3) can be estimated by means of the following formula:

$$
\begin{aligned}
\hat{H}= & H\left(\hat{c}, C_{T}\right)=\int_{[0,1]^{\beta}}\left(1-\sqrt{\frac{\hat{c}_{X Y}(u, v) \hat{c}_{X Z}(u, w)}{\hat{c}_{X Y Z}(u, v, w)}}\right)^{2} d C_{X Y Z}(u, v, w)= \\
& =\frac{1}{T} \sum_{t=1}^{T}\left(1-\sqrt{\frac{\hat{c}_{X Y}\left(\hat{F}_{X}\left(X_{T}\right), \hat{F}_{Y}\left(Y_{T}\right)\right) \hat{F}_{X Z}\left(\hat{F}_{X}\left(X_{T}\right), \hat{F}_{Z}\left(Z_{T}\right)\right)}{\hat{c}_{X Y Z}\left(\hat{F}_{X}\left(X_{T}\right), \hat{F}_{Y}\left(Y_{T}\right), \hat{F}_{Z}\left(Z_{T}\right)\right)}}\right)^{2}
\end{aligned}
$$

where $\hat{F}_{\text {. }}(\cdot)$ is the empirical form of marginal distribution $F .(\cdot)$. In addition, the densities of copulas are estimated by means of nonparametric methods.

Test statistics (comp. Bouezmarni et al. [12]) for $d_{1}=d_{2}=d_{3}=1$ is given by the formula:

$$
B R T=\frac{T k^{-3 / 2}}{\sigma}\left(4 \hat{H}-C_{1} T^{-1} k^{3 / 2}-\hat{B}_{1} T^{-1} k-\hat{B}_{2} T^{-1} k-\hat{B}_{3} T^{-1} k^{1 / 2}\right)
$$


where $C_{1}=2^{-3} \pi^{3 / 2}, \sigma=\sqrt{2}(\pi / 4)^{3 / 2}$ and

$$
\begin{gathered}
\hat{B}_{1}=-2^{-1} \pi+T^{-1} \sum_{t=1}^{T} \frac{\left(4 \pi \hat{G}_{t 1}\left(1-\hat{G}_{t 1}\right)\right)^{-\frac{1}{2}}\left(4 \pi \hat{G}_{t 2}\left(1-\hat{G}_{t 2}\right)\right)^{-\frac{1}{2}}}{\hat{c}_{X Y}\left(\hat{G}_{t 1}, \hat{G}_{t 2}\right)}, \\
\hat{B}_{2}=-2^{-1} \pi+T^{-1} \sum_{t=1}^{T} \frac{\left(4 \pi \hat{G}_{t 1}\left(1-\hat{G}_{t 1}\right)\right)^{-\frac{1}{2}}\left(4 \pi \hat{G}_{t 3}\left(1-\hat{G}_{t 3}\right)\right)^{-\frac{1}{2}}}{\hat{c}_{X Z}\left(\hat{G}_{t 1}, \hat{G}_{t 3}\right)} \\
\hat{B}_{3}=\pi^{-1 / 2} T^{-1} \sum_{t=1}^{T} \frac{1}{\sqrt{\hat{G}_{t 1}\left(1-\hat{G}_{t 1}\right)}} .
\end{gathered}
$$

The densities $\hat{c}_{X Y Z}, \hat{c}_{X Y}$ and $\hat{c}_{X Z}$ are estimated by means of Bernstein copulas. Under the null hypothesis the test statistic is distributed asymptotically standard normal. The null hypothesis is rejected for a given significance level $\alpha$ iff $B R T>z_{\alpha}$ holds true where $z_{\alpha}$ denotes the critical value given in the tables of standard normal distribution. Taking into account that the test statistic is asymptotically normal, the contributors advise in the case of a finite sample the calculation of $p$-values by means of bootstrap methods. Classic bootstrap methods referring to empirical distribution cannot be applied. Therefore, Paparoditis and Politis [37] suggested a local bootstrap method for nonparametric kernel estimators. They take into account the fact that the densities of the variables are conditional. This method was applied by Bouezmarni et al. [12] and Su and White [43]. The p-values can be determined for the samples $\left\{\left(X_{t}^{*}, Y_{t}^{*}, Z_{t}^{*}\right)\right\}_{t=1}^{T}$ generated by bootstraping under condition $d_{1}=d_{2}=d_{3}=1$ in the following steps:

1. In the first step $X_{t}^{*}$ is generated by means of the kernel estimator:

$$
\tilde{f}(x)=T^{-1} b^{-1} \sum_{t=1}^{T} f\left(\left(X_{t}-x\right) / b\right),
$$

where $f$ stands for the density of one dimensional distribution.

For $t=1, \ldots, T$ the values of $Y_{t}^{*}$ and $Z_{t}^{*}$ should be generated independently from conditional densities:

$$
\tilde{f}\left(y \mid X_{t}^{*}\right)=\frac{\sum_{s=1}^{T} f\left(\frac{\left(Y_{s}-y\right)}{b}\right) f\left(\frac{\left(X_{s}-X_{s}^{*}\right)}{b}\right)}{\sum_{s=1}^{T} f\left(\frac{\left(X_{t}-X_{s}^{*}\right)}{b}\right)}
$$




$$
\tilde{f}\left(z \mid X_{t}^{*}\right)=\frac{\sum_{s=1}^{T} f\left(\frac{\left(Z_{s}-z\right)}{b}\right) f\left(\frac{\left(X_{s}-X_{s}^{*}\right)}{b}\right)}{\sum_{s=1}^{T} f\left(\frac{\left(X_{t}-X_{s}^{*}\right)}{b}\right)} .
$$

2. For the generated sample test statistic $B R T^{*}$ should be established.

3. Steps 1-2 should be repeated $M$ times in order to receive $\left\{B R T_{j}^{*}\right\}_{j=1}^{M}$.

4. And finally the bootstrap p-value is given by

$$
p^{*}=\frac{1}{M} \sum_{j=1}^{M} \mathbf{1}_{\left\{B R T_{j}^{*}>B R T\right\}}
$$

\section{Data description and estimation of realized volatility}

The dataset (see Table 1) contains tick-by-tick transaction prices of five stocks from the Vienna Stock Exchange from 2 January 2006 to 9 November 2011 (1454 daily observations). The selected stocks are Andritz AG (ANDR), Erste Group Bank AG (EBS), OMV AG (OMV), Telekom Austria AG (TKA) and Voestalpine AG (VOE). For these companies, descriptive statistics of time series of returns, realized volatility and trading volume were computed. They are presented below.

\section{Daily stock returns}

We computed daily stock returns at close and multiplied them by 100 . The table below presents descriptive statistics of logarithimic stock returns $r_{t}$ and the results of Ljung-Box and Jarque-Bera tests ( $p$-values).

Table 1

Descriptive statistics of stock returns

\begin{tabular}{|l|c|c|c|c|c|c|}
\cline { 2 - 7 } \multicolumn{1}{c|}{} & mean & $\begin{array}{c}\text { std } \\
\text { deviation }\end{array}$ & skewness & kurtosis & $\begin{array}{c}\text { p-value } \\
\text { L-B }\end{array}$ & $\begin{array}{c}\text { p-value } \\
\text { J-B }\end{array}$ \\
\hline ANDRITZ & 0.073 & 2.985 & 0.099 & 8.617 & 0.001 & 0.001 \\
\hline ERSTEBANK & -0.082 & 3.510 & -0.154 & 7.902 & 0.013 & 0.001 \\
\hline OMV & -0.049 & 2.626 & -0.459 & 7.393 & 0.476 & 0.001 \\
\hline TKA & -0.058 & 2.202 & -0.763 & 13.049 & 0.007 & 0.001 \\
\hline VOEST & 0.007 & 3.280 & -0.157 & 7.289 & 0.872 & 0.001 \\
\hline
\end{tabular}

Source: own calculations 
The Testing of Causal Stock Returns-Trading Volume Dependencies...

The results confirmed the stylized facts about stock returns $r_{t}$. The departure from normality is reflected in kurtosis and skewness. The null hypothesis about normality is rejected for all companies under study. In three cases we observe significant autocorrelation.

\section{Realized volatility}

As a proxy of volatility in empirical investigations squared returns or absolute returns are used. For high frequency data a better alternative is realized volatility.

Suppose that $p_{i, t}$ is the logarithm of price (expressed in \%) $m$ times in day $t$ in equal time intervals. The estimator of realized volatility is defined as

$$
R V_{t}=\sum_{i=1}^{m}\left(p_{i, t}-p_{i-1, t}\right)^{2}=\sum_{i=1}^{m} r_{i, t}^{2}
$$

Under some regularity conditions $R V_{t}$ is a consistent estimator of integrated volatility $I V_{t}=\int_{t}^{t+1} \sigma_{s}^{2} d s$, where in the stochastic differential equation $d p_{t}=\mu_{t} d t+$ $+\sigma_{t} d W_{t}$ for logarithmic prices, the variable $\sigma_{t}$ stands for volatility $\mu_{t}$ is drift and $W_{t}$ is the Wiener process. Unfortunately in most cases $R V_{t}$ is a biased estimator because of autocorrelation in intraday data, caused by a microstructure noise effect. The autocorrelation increases with rising frequency. In order to reduce the bias and mean squared error one can include covariances. Another solution is the choice of optimal sampling frequency (Bandi and Russell [3, 4, 6]), Zhang et al. [46], Hansen and Lunde [26, 27] and Oomen [36]).

Barndorff-Nielsen et al. $[8,9,10]$ have introduced the class of realized kernel estimators. A survey of realized variance estimators can be found in Bandi and Russel [4] and McAleer and Medeiros [34].

In this paper we use a Newey-West estimator based on the Bartlett kernel for daily realized volatility (Hansen and Lunde [26]):

$$
R V_{t}^{N W}=\sum_{i=1}^{m} r_{i, t}^{2}+2 \sum_{k=1}^{q}\left(1-\frac{k}{q+1}\right) \sum_{i=1}^{\mathrm{m}-\mathrm{k}} r_{i, t} r_{i+k, t} .
$$

This estimator has many advantages. However, it does not take into account volatility between the close of a session and the opening of the session next day. Therefore, it is necessary to add to $R V_{t}^{N W}$ a square of return computed for the price at close and the price at open denoted by $r_{C O t}$.

Hansen and Lunde [26] introduced the realized volatility estimation method for a whole day. In order to minimize the mean square error the linear combination of $r_{Z O t}^{2}$ and $R V_{t}^{N W}$ was taken into account. The optimal, conditionally unbiased, minimum variance estimator of realized volatility in the class of linear estimators is:

$$
\tilde{R V_{t}}=\omega_{1} r_{C O t}^{2}+\omega_{2} R V_{t}^{N W},
$$


where

$$
\omega_{1}=(1-\varphi) \frac{\mu_{0}}{\mu_{1}}, \quad \omega_{2}=\varphi \frac{\mu_{0}}{\mu_{2}}
$$

and

$$
\varphi=\frac{\mu_{2}^{2} \eta_{1}^{2}-\mu_{1} \mu_{2} \eta_{12}}{\mu_{2}^{2} \eta_{1}^{2}-\mu_{1}^{2} \eta_{2}^{2}-2 \mu_{1} \mu_{2} \eta_{12}}
$$

and $r_{C O t}$ is the close-to-open log-return.

The parameter $\varphi$ gives a relative importance factor that indirectly stands for a portion of the volatility observed during the session. The parameters in the equations above are computed as follows:

$$
\begin{gathered}
\mu_{0}=E\left(r_{C O t}^{2}+R V_{t}^{N W}\right), \mu_{1}=E\left(r_{C O t}^{2}\right), \mu_{2}=E\left(R V_{t}^{N W}\right) \\
\eta_{1}^{2}=\operatorname{var}\left(r_{C O t}^{2}\right), \eta_{2}^{2}=\operatorname{var}\left(R V_{t}^{N W}\right), \eta_{12}=\operatorname{cov}\left(r_{C O t}^{2}, R V_{t}^{N W}\right) .
\end{gathered}
$$

Based on high frequency data, by applying these formulae to opening and closing prices the time series of realized volatility were derived. In the NeweyWest estimator the time lags were chosen in order to minimize the mean square error. In addition, for the companies listed on Vienna Stock Exchange an optimal frequency parameter $m$ was estimated. The estimators of realized volatility and volatility computed as squared daily log-returns are presented in Figure 2 (for stock ANDR):
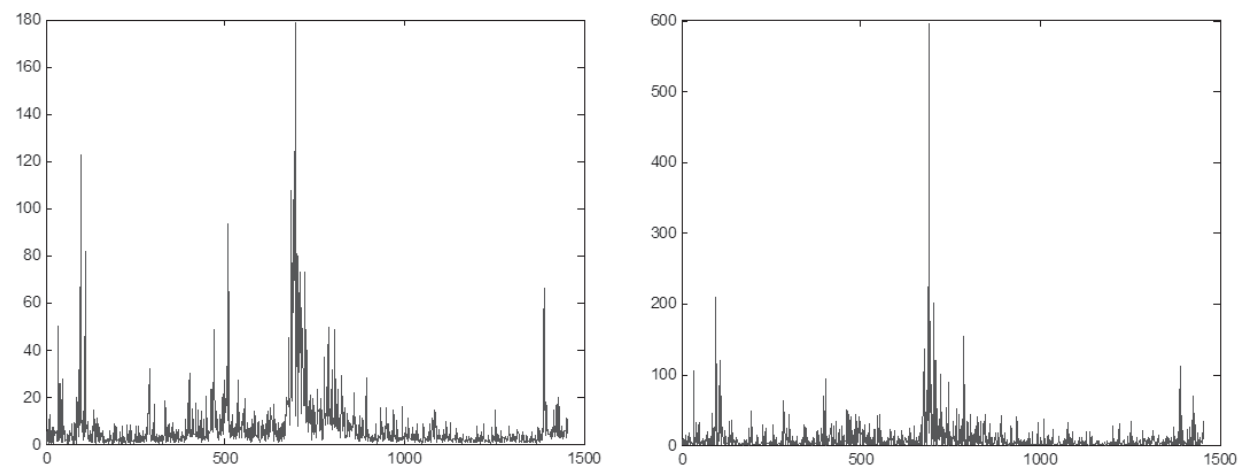

Figure 2. Realized volatility (left) and squared daily log-returns (right) of ANDR Source: own calculations based on data from Vienna Stock Exchange 
The Testing of Causal Stock Returns-Trading Volume Dependencies...

We applied a logarithmic transformation to the realized volatility series. Descriptive statistics are presented in Table 2.

Table 2

Descriptive statistics of logarithmic realized volatility

\begin{tabular}{|l|c|c|c|c|c|c|}
\cline { 2 - 7 } \multicolumn{1}{c|}{} & mean & $\begin{array}{c}\text { std } \\
\text { deviation }\end{array}$ & skewness & kurtosis & $\begin{array}{c}\text { p-value } \\
\text { L-B }\end{array}$ & $\begin{array}{c}\text { p-value } \\
\text { J-B }\end{array}$ \\
\hline ANDRITZ & 1.351 & 1.061 & 0.289 & 3.398 & 0.000 & 0.001 \\
\hline ERSTEBANK & 1.554 & 1.117 & 0.423 & 2.844 & 0.000 & 0.001 \\
\hline OMV & 1.158 & 0.930 & 0.565 & 3.575 & 0.000 & 0.001 \\
\hline TKA & 0.822 & 0.987 & 0.392 & 3.146 & 0.000 & 0.001 \\
\hline VOEST & 1.717 & 0.916 & 0.483 & 3.334 & 0.000 & 0.001 \\
\hline
\end{tabular}

Source: own calculations

The results presented above indicate that despite the application of the logarithmic transformation the series are not normally distributed. We observe that all of the series are positively skewed. Therefore, we removed the deterministic trend from the time series. The series adjusted in this way are denoted as $\ln R V_{t}$. Since in the next sections VAR models are used, we checked stationarity by an augmented Dickey-Fuller test for unit root:

- test $1(\mathrm{ADF})$ :

$$
\begin{gathered}
H_{0}: y_{t}=y_{t-1}+\sum_{j=1}^{k} \beta_{j} \Delta y_{t-j}+\varepsilon_{t}, \\
H_{1}: y_{t}=\alpha y_{t-1}+\sum_{j=1}^{k} \beta_{j} \Delta y_{t-j}+\varepsilon_{t}, \text { where } \alpha<1 .
\end{gathered}
$$

- test 2 (ADF with drift):

$$
\begin{gathered}
H_{0}: y_{t}=y_{t-1}+\sum_{j=1}^{k} \beta_{j} \Delta y_{t-j}+\varepsilon_{t}, \\
H_{1}: y_{t}=\gamma+\alpha y_{t-1}+\sum_{j=1}^{k} \beta_{j} \Delta y_{t-j}+\varepsilon_{t}, \text { where } \alpha<1 .
\end{gathered}
$$

The values of lag $k$ are selected upon information criteria AIC and BIC. Table 3 presents the results of testing. 
Table 3

Results of unit root testing for $\ln R V_{t}$

\begin{tabular}{|l|c|c|}
\hline \multicolumn{2}{|c|}{ Companies listed in ATX index } \\
\hline \multicolumn{1}{|c|}{ test 1} & test 2 \\
\hline ANDR & -14.07 & -14.07 \\
\hline EBS & -10.52 & -10.51 \\
\hline OMV & -12.75 & -12.75 \\
\hline TKA & -12.23 & -12.23 \\
\hline VOE & -11.40 & -11.40 \\
\hline
\end{tabular}

Source: own calculations

In all cases the null hypothesis of unit root is rejected, so series $\ln R V_{t}$ can be used in VAR models.

\section{Trading Volume}

The daily trading volume is calculated as the sum of trading volumes corresponding to each transaction from a whole given day. In Table 4 below we present the descriptive statistics of log-volume.

Table 4

Descriptive statistics of log-volume

\begin{tabular}{|l|c|c|c|c|}
\cline { 2 - 5 } \multicolumn{1}{c|}{} & mean & $\begin{array}{c}\text { std. } \\
\text { deviation }\end{array}$ & kurtosis & skewness \\
\hline ANDR & 11.915 & 0.569 & 0.146 & 3.322 \\
\hline EBS & 13.703 & 0.584 & 0.198 & 2.909 \\
\hline OMV & 13.393 & 0.489 & 0.234 & 3.523 \\
\hline TKA & 13.883 & 0.512 & 0.084 & 2.974 \\
\hline VOE & 13.204 & 0.524 & 0.210 & 3.354 \\
\hline
\end{tabular}

Source: own calculations

Only in the case of TKA, can the null hypothesis of normality ( $p$-values of Jarque - Bera test, not reported here) not be rejected. We filtered the log-volume from the deterministic trend and calendar effects. Before using VAR models we performed a unit root test (Tab. 5). 
Table 5

Results of unit root testing of filtered log-volume

\begin{tabular}{|l|c|c|}
\cline { 2 - 3 } \multicolumn{1}{c|}{} & test 1 & test 2 \\
\hline ANDR & -0.04 & -11.72 \\
\hline EBS & -0.11 & -10.40 \\
\hline OMV & -0.05 & -11.89 \\
\hline TKA & -0.12 & -15.28 \\
\hline VOE & -0.20 & -8.85 \\
\hline
\end{tabular}

Source: own calculations

By means of an ADF test with drift we rejected the null hypotesis of unit root in all cases. In the next sections we consider two types of trading volume: expected and unexpected. Unexpected trading volume $\left(\widetilde{\ln V}_{t}\right)$ is the part of the total volume that can't be forecasted and it is generated by the random process of new pieces of information coming to the market. An expected trading volume $\left(\ln V_{t}\right)$ can be forecasted and we used fitted values of ARMA models to describe it. An unexpected trading volume is given by the residuals from ARMA models.

\section{Empirical results and their analysis}

In this section by means of nonparamteric Bernstein copulas we analyse pairwise nonlinear causality between prices, trading volume and realized volatility.

\section{Causal price-trading volume relations}

To test the linear Granger causality we applied a bivariate $\operatorname{VAR}(\boldsymbol{k})$ model for $\boldsymbol{P}_{t}=\left(X_{t} Y_{t}\right)^{\prime}:$

$$
\boldsymbol{P}_{\boldsymbol{t}}=\Phi_{0}+\sum_{i=1}^{k} \Phi_{i} \boldsymbol{P}_{\boldsymbol{t}-\boldsymbol{i}}+\varepsilon_{\boldsymbol{t}}
$$

where $\Phi_{0}$ is the vector of intercepts and $\Phi_{i}=\left(\begin{array}{ll}\phi_{11, i} & \phi_{12, i} \\ \phi_{21, i} & \phi_{22, i}\end{array}\right)$ is the matrix of parameters corresponding to $\boldsymbol{P}_{t-i}$.

The null hypothesis that $X$ does not Granger cause $Y$ is equivalent to $\phi_{21, i}=0$ for $i=1, \ldots, k$. We tested the null using $F$-statistics of the form:

$$
F=\frac{S S E_{0}-S S E}{S S E} \cdot \frac{T-2 k-1}{k}
$$


where $S S E_{0}$ is the sum of the squared residuals of the restricted regression $\left(\phi_{21, i}=0\right)$ and $S S E$ is the sum of the squared residuals of the unrestricted model. The number of observations is denoted by $T$. Under the null the statistics presented above is asymptotically $F$ distributed with $k$ and $T-2 k-1$ the degrees of freedom. The choice of $k$ is based on the AIC and the BIC criteria. The proper number $k$ of time lags guarantees that residuals are uncorrelated.

In order to test the nonlinear causality we used the BRT statistics described in the previous section, applied to residuals from VAR models. Using this method we can be sure that we tested only nonlinear relations. When estimating the Bernstein copulas we took the bandwidth $k$ as the integer part of $2 \sqrt{T}$. We computed the $p$-values of the test with 200 bootstrap samples. Below we used the notations $X \nrightarrow Y$ in order to describe the null hypothesis: $X$ does not Granger cause $Y$.

\section{Realized volatility and expected trading volume}

The linear, causal relations between realized volatility and expected trading volume were tested with the VAR model described above. To test the presence of nonlinear relations we formulated the following null hypotheses

$$
H_{0}: f\left(\ln R V_{t} \mid \ln R V_{t-1}, \ln V_{t-1}\right)=f\left(\ln R V_{t} \mid \ln R V_{t-1}\right)
$$

and

$$
H_{0}: f\left(\ln V_{t} \mid \ln V_{t-1}, \ln R V_{t-1}\right)=f\left(\ln V_{t} \mid \ln V_{t-1}\right)
$$

The first of them is equivalent to $H_{0}: \ln V_{t} \nrightarrow \ln R V_{t}$ and the second to $H_{0}: \ln R V_{t} \nrightarrow \ln V_{t}$. The Table 6 below summarizes the results of testing ( $p$-values).

Table 6

Results of testing for the pair realized volatility - expected trading volume

\begin{tabular}{|l|c|c|c|c|}
\hline \multicolumn{1}{|c|}{$H_{\mathbf{0}}$} & \multicolumn{2}{c|}{$\ln R V_{\boldsymbol{t}} \nrightarrow \ln V_{\boldsymbol{t}}$} & \multicolumn{2}{c|}{$\ln V_{\boldsymbol{t}} \nrightarrow \ln R V_{\boldsymbol{t}}$} \\
\hline test & linear & BRT & linear & BRT \\
\hline ANDR & 0.000 & 0.495 & 0.300 & 0.170 \\
\hline EBS & 0.000 & 0.120 & 0.282 & 0.620 \\
\hline OMV & 0.000 & 0.035 & 0.206 & 0.140 \\
\hline TKA & 0.000 & 0.020 & 0.000 & 0.620 \\
\hline VOE & 0.000 & 0.045 & 0.022 & 0.745 \\
\hline
\end{tabular}

Source: own calculations 
In all cases there is linear causality running from realized volatility to expected trading volume. Causality in the opposite direction is detected only in the case of TKA and VOE. In addition, there is nonlinear causality running from $\ln R V_{t}$ to $\ln V_{t}$ for three stocks (OMV, TKA and VOE).

\section{Realized volatility and unexpected trading volume}

We replace $\ln V_{t}$ with $\widetilde{\ln V}_{t}$ and estimate VAR models and the required copulas again. The set of hypotheses under study is:

$$
H_{0}: \ln R V_{t} \nrightarrow \widetilde{\ln V}_{t} \text { against } H_{1}: \ln R V_{t} \rightarrow \widetilde{\ln V_{t}}
$$

and

$$
H_{0}: \widetilde{\ln V}_{t} \nrightarrow \ln R V_{t} \text { against } H_{1}: \widetilde{\ln }_{t} \rightarrow \ln R V_{t}
$$

Table 7

\begin{tabular}{|c|c|c|c|c|}
\hline $\boldsymbol{H}_{0}$ & \multicolumn{2}{|c|}{$\ln R V_{t} \nrightarrow \widetilde{\ln V_{t}}$} & \multicolumn{2}{|c|}{$\widetilde{\ln V}_{t} \nrightarrow \ln R V_{t}$} \\
\hline test & linear & BRT & linear & BRT \\
\hline ANDR & 0.000 & 1.000 & 0.086 & 1.000 \\
\hline EBS & 0.000 & 0.940 & 0.081 & 1.000 \\
\hline OMV & 0.023 & 1.000 & 0.128 & 1.000 \\
\hline TKA & 0.356 & 1.000 & 0.000 & 0.990 \\
\hline VOE & 0.000 & 0.845 & 0.000 & 1.000 \\
\hline
\end{tabular}

Results of testing for the pair realized volatility - unexpected trading volume

Source: own calculations

In all cases (see Table 7) there is no nonlinear causal relationship in either directions. We observed linear causality from realized volatility to unexpected trading volume in four cases (the only exception is TKA). Causality in the opposite direction was detected in two cases with a significance level 0.05 and four cases with 0.1 .

\section{Stock returns and expected trading volume}

The hypotheses

$$
\begin{aligned}
& H_{0}: r_{t} \nrightarrow \ln V_{t}, \\
& H_{1}: r_{t} \rightarrow \ln V_{t},
\end{aligned}
$$


in terms of conditional densities are formulated as follows:

$$
\begin{aligned}
& H_{0}: f\left(\ln V_{t} \mid \ln V_{t-1}, r_{t-1}\right)=f\left(\ln V_{t} \mid \ln V_{t-1}\right), \\
& H_{1}: f\left(\ln V_{t} \mid \ln V_{t-1}, r_{t-1}\right) \neq f\left(\ln V_{t} \mid \ln V_{t-1}\right) .
\end{aligned}
$$

The opposite direction of causal dependency has the form:

$$
\begin{aligned}
& H_{0}: f\left(r_{t} \mid, r_{t-1}, \ln V_{t-1}\right)=f\left(r_{t} \mid r_{t-1}\right) \\
& H_{1}: f\left(r_{t} \mid, r_{t-1}, \ln V_{t-1}\right) \neq f\left(r_{t} \mid r_{t-1}\right),
\end{aligned}
$$

\begin{tabular}{|c|c|c|c|c|}
\hline $\boldsymbol{H}_{0}$ & \multicolumn{2}{|c|}{$r_{t} \nrightarrow \ln V_{t}$} & \multicolumn{2}{|c|}{$\ln V_{t} \nrightarrow r_{t}$} \\
\hline test & linear & BRT & linear & BRT \\
\hline ANDR & 0.004 & 0.000 & 0.186 & 0.985 \\
\hline EBS & 0.000 & 0.000 & 0.266 & 0.070 \\
\hline OMV & 0.001 & 0.000 & 0.449 & 0.715 \\
\hline TKA & 0.000 & 0.000 & 0.945 & 0.115 \\
\hline VOE & 0.000 & 0.000 & 0.945 & 0.140 \\
\hline
\end{tabular}

Table 8 below presents the p-values of tests conducted.

Table 8

Results of causality testing for stock returns and expected trading volume

Source: own calculations

We observed linear and nonlinear causality from stock returns to expected trading volume. A relationship in the opposite direction doesn't occur. The only exception is nonlinear causality for EBS (at 0.1 significance level).

\section{Stock returns and unexpected trading volume}

First we estimated a bivariate VAR model for pair $r_{t}-\widetilde{\ln V}_{t}$. As in the previous sections we used an empricial distribution function in order to transform residuals from this model. The respective hypotheses are:

$$
H_{0}: r_{t} \nrightarrow \widetilde{\ln V}_{t} \text { against } H_{1}: r_{t} \rightarrow \widetilde{\ln V}_{t}
$$

and

$$
H_{0}: \widetilde{\ln }_{t} \nrightarrow r_{t} \text { against } H_{1}: \widetilde{\ln V}_{t} \rightarrow r_{t}
$$


The Testing of Causal Stock Returns-Trading Volume Dependencies...

Table 9

Results of causality testing for stock returns and unexpected trading volume

\begin{tabular}{|l|c|c|c|c|}
\hline \multicolumn{1}{|c|}{$\boldsymbol{H}_{\mathbf{0}}$} & \multicolumn{2}{|c|}{$\boldsymbol{r}_{\boldsymbol{t}} \nrightarrow \widetilde{\boldsymbol{m V}}_{\mathbf{t}}$} & \multicolumn{2}{c|}{$\widetilde{\boldsymbol{m} V_{\mathbf{t}}} \nrightarrow \boldsymbol{r}_{\boldsymbol{t}}$} \\
\hline test & linear & BRT & linear & BRT \\
\hline ANDR & 0.025 & 0.105 & 0.531 & 0.760 \\
\hline EBS & 0.000 & 0.010 & 0.474 & 0.990 \\
\hline OMV & 0.021 & 0.855 & 0.402 & 1.000 \\
\hline TKA & 0.000 & 0.840 & 0.909 & 0.820 \\
\hline VOE & 0.000 & 0.890 & 0.909 & 0.865 \\
\hline
\end{tabular}

Source: own calculations

The structure of dependence (see Table 9) is very clear. There is no nonlinear relationship in either direction (with the exception of EBS stock). We observed only linear causality from returns to unexpected trading volume, so a knowledge of prices from previous days can be helpful in forecasting unexpected trading volume when using linear models.

\section{Conclusions}

The authors applied the test set out by Bouezmarni et al. [12] for conditional independence between two vector processes conditional one on another. The test applied is based on computer support and it is easy to conduct. The main reason for this is that it does not involve a weighting function in the test statistic. In addition, it can be applied in general settings since there is no restriction on the dimension of the data. To apply this test, only a bandwidth for the nonparametric copula is needed. The causal price-volume relationships were investigated for selected stocks traded on the Vienna Stock Exchange by using high frequency data. To detect linear causality classical vector autoregressive models were used. The nonlinear form of relationships were examined using a test based on nonparametric copulas.

The stock return volatility was computed using realized volatility estimators including changes in prices for non-trading hours. There are some clear patterns of causal relationships between stock returns, realized volatility and expected and unexpected trading volume. There is linear causality running from realized volatility to expected trading volume, and a lack of nonlinear causal dependence in the opposite direction. When unexpected trading volume is used, we observe (with one exception) linear causality for pair volatility-trading volume in both 
directions and a lack of nonlinear causality. When regarding the pair stock returns and trading volume the conclusions depend on the part of trading volume used. There is a strong a linear and nonlinear causality from stock returns to expected trading volume, and a lack of such a relationship in the opposite direction. So a knowledge of past stock returns can improve forecasts of expected trading volume. When regarding unexpected trading volume, we conclude that there is only a linear, causal relationship from stock returns to unexpected trading volume. Neither linear nor nonlinear causal relations from unexpected trading volume to stock returns are detected.

\section{References}

[1] Abhyankar A., Linear and non-linear Granger causality: Evidence from the U.K. stock index futures market, "Journal of Futures Markets" 1998, vol. 18, pp. 519-540.

[2] Asimakopoulos I., Ayling D., Mahmood W.M., Non-linear Granger causality in the currency futures returns, "Economics Letters" 2000, vol. 68, pp. 25-30.

[3] Bandi F.M., Russell J.R., Separating microstructure noise from volatility, "Journal of Financial Economics" 2006, vol. 79(3), pp. 655-692.

[4] Bandi F.M., Russell J.R., Microstructure noise, realized variance, and optimal sampling, "Review of Economic Studies" 2008a, vol. 75(2), pp. 339-369.

[5] Bandi F.M., Russell J.R., Yang C., Realized volatility forecasting and option pricing, "Journal of Econometrics" 2008b, vol. 147(1), pp. 34-46.

[6] Bandi F.M., Russell J.R., Market microstructure noise, integrated variance estimators, and the accuracy of asymptotic approximations, "Journal of Econometrics" 2011, vol. 160(1), pp. 145-159.

[7] Baek E., Brock W., A general test for Granger causality: Bivariate model, Technical Report 1992, Iowa State University and University of Wisconsin, Madison.

[8] Barndorff-Nielsen O.E., Hansen P.R., Lunde A., Shephard N., Regular and modified kernel-based estimators of integrated variance: The case with independent noise?, "Economics Papers" 2004-W28, Economics Group, Nuffield College, University of Oxford.

[9] Barndorff-Nielsen O.E., Hansen P.R., Lunde A., Shephard N., Designing realized kernels to measure the ex-post variation of equity prices in the presence of noise, "Econometrica" 2008 , vol. 76(6), pp. 1481-1536.

[10] Barndorff-Nielsen O.E., Hansen P.R., Lunde A., Shephard N., Realized kernels in practice: Trades and quotes, "Econometrics Journal" 2009, vol. 12, pp. 1-32.

[11] Bollerslev T., Jubinski D., Equity trading volume volatility: latent information arrivals and common long-run dependencies, "Journal of Business \& Economic Statistics" 1999, vol. 17, pp. 9-21. 
The Testing of Causal Stock Returns-Trading Volume Dependencies...

[12] Bouezmarni T., Rombouts J.V.K., Taamouti A., A nonparametric copula based test for conditional independence with applications to Granger causality, "Journal of Business and Economic Statistics" 2012, to appear.

[13] Clark P.K., A subordinated stochastic process model with finite variance for speculative prices, "Econometrica“" 1973, vol. 41, pp. 135-156.

[14] Copeland T.E., A model of asset trading under the assumption of sequential information arrival, "Journal of Finance" 1976, vol. 31, pp. 1149-1168.

[15] Diks C., Panchenko V., A Note on the Hiemstra-Jones Test for Granger Noncausality, "Studies in Nonlinear Dynamics \& Econometrics" 2005, vol. 9(2), Article 4.

[16] Diks C., Panchenko V., A new statistic and practical guidelines for nonparametric Granger causality testing, "Journal of Economic Dynamics and Control", vol. 30(9-10), pp. 1647-1669.

[17] Epps T.W., Epps M.L., The stochastic dependence of security price changes and transaction volumes: implications for the mixture-of-distribution bypothesis, "Econometrica" 1976, vol. 44, pp. 305-321.

[18] Fleming J., Kirby C., Long memory in volatility and trading volume, "Journal of Banking \& Finance” 2011, vol. 35(7), pp. 1714-1726.

[19] Gervais S., Kaniel R., Mingelgrin D., The high-volume return premium, "The Journal of Finance" 2001, vol. 56, pp. 877-919.

[20] Granger C.W.J., Investigating causal relations by econometric models and cross-spectral methods, "Econometrica" 1969, vol. 37 (3), pp. 424-438.

[21] Granger C.W.J., Newbold P., Spurious regression in econometrics, "Journal of Econometrics" 1974, vol. 2, pp. 111-120.

[22] Gurgul H., Lach Ł., Liniowa i nieliniowa przyczynowość dla spółek zindeksu $D A X$, „Badania Operacyjne i Decyzje” 2009, vol. 3, pp. 27-46.

[23] Gurgul H., Majdosz P., Mestel R., Price - volume relations of DAX companies, "Financial Markets and Portfolio Management" 2007, vol. 21, pp. 353-379.

[24] Gurgul H., Majdosz P., Mestel R., Joint Dynamics of Prices and Trading Volume on the Polish Stock Market, "Managing Global Transitions" 2005, vol. 3(2), pp. 139-156.

[25] Gurgul H., Wójtowicz T., Stopy zwrotu a wielkość obrotów na GPW w Warszawie, "Folia Oeconomica Cracoviensia" 2008, vol. 49-50, pp. 31-45.

[26] Hansen P.R., Lunde A., A realized variance for the whole day based on intermittent high-frequency data, "Journal of Financial Econometrics" 2005, vol. 3(4), pp. 525-554.

[27] Hansen P.R., Lunde A., Realized variance and market microstructure noise, "Journal of Business and Economic Statistics" 2006, vol. 24(2), pp. 127-161.

[28] Harris L., Cross-security tests of the mixture of distributions bypothesis, "Journal of Financial and Quantitative Analysis" 1986, vol. 21, pp. 39-46.

[29] Hiemstra C., Jones J.D., Testing for linear and nonlinear Granger causality in the stock price - volume relation, "Journal of Finance" 1994, vol. 49, pp. 1639-1664. 
[30] Karpoff J.M., The Relation between Price Changes and Trading Volume: A Survey, "Journal of Financial and Quantitative Analysis" 1987, vol. 22, pp. 109-126.

[31] Lee B.S., Rui O.M., The dynamic relationship between stock returns and trading volume: Domestic and cross-country evidence, "Journal of Banking and Finance" 2002, vol. 26, pp. 51-78.

[32] Linton O., Gozalo P., Conditional Independence Restrictions: Testing and Estimation, Discussion Paper 1997, Cowles Foundation for Research in Economics, Yale University.

[33] Luu J.C., Martens M., Testing the mixture-of-distributions bypothesis using "realized" volatility, "Journal of Futures Markets" 2003, vol. 23(7), pp. 661-679.

[34] McAleer M., Medeiros M., Realized volatility: A review, "Econometric Reviews" 2008, vol. 27, pp. 10-45.

[35] Nielsen M.Ø., Shimotsu K., Determining the cointegrating rank in nonstationary fractional systems by the exact local Whittle approach, "Journal of Econometrics" 2007, vol. 141, pp. 574-96.

[36] Oomen R.C.A., Properties of realized variance under alternative sampling schemes, "Journal of Business and Economic Statistics" 2006, vol. 24, pp. 219-237.

[37] Paparoditis E., Politis D., The Local Bootstrap for Kernel Estimators under General Dependence Conditions, "Annals of the Institute of Statistical Mathematics" 2000, vol. 52, pp. 139-159.

[38] Phillips C.P., Understanding the spurious regression in econometrics, "Journal of Econometrics" 1986, vol. 33, pp. 311-40.

[39] Robinson P.M., Yajima Y., Determination of cointegrating rank in fractional systems, "Journal of Econometrics" 2002, vol. 106 (2), pp. 217-241.

[40] Rossi, E., de Magistris P.S., Long memory and tail dependence in trading volume and volatility, "CREATES Research Paper" No. 2009-30.

[41] Sims C.A., Stock J.H., Watson M.W., Inference in linear time series models with some unit roots, "Econometrica" 1990, vol. 58, pp. 133-144.

[42] Su L., White H., Testing Conditional Independence via Empirical Likelibood, Discussion Paper 2003, University of California San Diego.

[43] Su L., White H., A nonparametric Hellinger Metric test for Conditional Independence, "Econometric Theory" 2008, vol. 24, pp. 1-36.

[44] Tauchen G.E.; Pitts M., The price variability - volume relationship on speculative markets, "Econometrica" 1983, vol. 51, pp. 485-505.

[45] Toda H.Y., Yamamoto T., Statistical inference in vector autoregressions with possibly integrated processes, "Journal of Econometrics" 1995, vol. 66, pp. 225-250.

[46] Zhang L., Mykland P., Aït-Sahalia Y., A tale of two time scales: determining integrated volatility with noisy high-frequency data, "Journal of the American Statistical Association” 2005, vol. 100, pp. 1394-1411. 